\title{
Le concept reproducteur
}

James Griesemer

$>$ Ce qui relève de l'épigénétique dépend de ce que l'on considère comme génétique. Le weismannisme, qui a dominé la biologie au $x x^{e}$ siecle et qui oppose la continuité génétique à la discontinuité somatique, aboutit à une définition de l'épigénétique qui englobe de façon excessive tous les mécanismes de l'hérédité non expliquée par le gène (beyond the genes). En fait, une autre vision théorique peut être proposée, le « concept reproducteur», qui facilite l'analyse des multiples systèmes de l'hérédité en évitant que toute forme d'hérédité non génétique soit obligatoirement taxée d'épigénétique. <

\section{Une nouvelle vision de l'épigénétique}

Le weismannisme, qui défend l'opposition entre la continuité du germen ${ }^{1}$ et la discontinuité du soma (Figure 1) [1-3], a dominé la biologie au xx siècle. Si l'on sait surtout de cette doctrine qu'elle rejette l'idée d'une hérédité des caractères acquis, les aboutissants du weismannisme sont plus subtils que cela $[4,5]$ : de fait, le weismannisme explore les relations entre cellules germinales et somatiques, mais aussi entre génotype et phénotype, hérédité et développement, évolution et sélection. Dans cette théorie, toute causalité (sauf celle due à des facteurs environnementaux, ignorée à l'échelle de la cellule ou de l'organisme) a son origine dans le germen, ou les gènes; le corps, ou le phénotype, constitue quant à lui une impasse causale. La vision weismannienne a également été étendue à l'échelon moléculaire, en raison de son isomorphisme avec le dogme central de Crick (Figure 1) ${ }^{2}$ : ces concepts sont les fondements de notre représentation de ce qui est génétique, et par conséquent de ce qui est épigénétique [6].

Adaptation et traduction: Emmanuelle Chollet et Michel Morange. D'après Griesemer J. What's « epi » about epigenetics? Ann NY Acad Sci 2002; 981: 97-100.

${ }^{1}$ Le germen correspond à l'ensemble des cellules germinales, par opposition au soma constitué du reste des cellules d'un organisme (note du traducteur).

${ }^{2}$ C'est-à-dire l'existence d'un sens unique de transmission de l'information $\mathrm{ADN} \rightarrow \mathrm{ARN} \rightarrow$ protéines, sans possibilité de retour d'information des protéines vers le génome (note du traducteur).

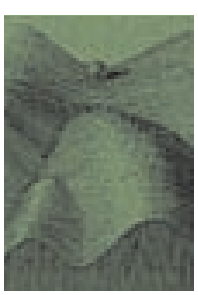

Le curieux pouvoir d'une vision théorique est de s'ancrer dans la pratique scientifique, y compris chez ceux qui la rejettent: il est probable que c'est Department of philosophy, University of California, One Shields Avenue, Davis, CA 95616-8673, États-Unis. jrgriesemer@ucdavis.edu

ce qui se passe pour la recherche en épigénétique. On a ainsi souvent recours au weismannisme dès qu'il s'agit d'expliquer un phénomène épigénétique, même si les données de la recherche montrent qu'il s'agit là d'une théorie erronée. Prenons l'exemple de la méthylation d'une cytosine, qui peut entraîner un changement de séquence via la désamination de la 5 -méthylcytosine et la formation subséquente de thymidine [7]: le weismannisme, comme d'ailleurs le dogme central de la biologie moléculaire, sont mis en échec, puique la causalité ne s'observe plus seulement dans le sens unique gènes $\rightarrow$ protéines, ou génotype $\rightarrow$ phénotype. De plus, en partant du principe que la désamination ne dépend pas d'une quelconque variation dans la séquence des gènes codant pour les enzymes de méthylation, la causalité n'est alors décidément pas imputable aux gènes, même indirectement via une modification de séquences enzymatiques.

La difficulté à articuler une nouvelle vision théorique qui reconnaîtrait la toute nouvelle épigénétique tout en préservant la distinction entre weismannisme et lamarckisme est probablement due à ce caractère bien ancré du weismannisme. En effet, il est typique en sciences de mettre en compétition plusieurs modèles pour aboutir à une «vérité » biologique : si deux modèles diffèrent dans leurs aspects fondamentaux, on estime qu'ils ne peuvent être tous deux vrais, et la démarche essentielle est alors d'élaborer des tests empiriques permettant d'opter pour l'un ou l'autre d'entre eux. Une alternative existe, qui est de considérer les modèles de façon non nécessairement compétitive : plusieurs visions peuvent 
être nécessaires pour orienter l'évaluation des modèles et produire ainsi des théories solides.

Cet article ne propose donc pas de substituer une nouvelle théorie au weismannisme, mais plutôt de lui trouver un complément capable d'augmenter les chances de succès des théories empiriques. II reprend les questions posées par les phénomènes épigénétiques, identifie en quoi le weismannisme peut, en tant que vision théorique, être inadéquat pour les expliquer, et suggère, enfin, la manière de formuler une nouvelle théorie. Il importe d'abord de rappeler en quoi le weismannisme a fait de l'hérédité et du développement deux processus biologiques distincts et séparés (voir aussi [8]), et de montrer que, à la lumière des observations épigénétiques, l'un et l'autre peuvent en fait être vus comme deux phénomènes entrelacés.

\section{Hérédité versus développement}

L'opposition entre hérédité et développement relève probablement plus d'une idée développée à la paillasse que d'une véritable dichotomie originelle entre deux disciplines, la génétique et la biologie du développement. Reprenons le diagramme synthétisant le weismannisme (Figure 1) et son isomorphisme avec le dogme central de la biologie moléculaire: selon Crick, «une fois que l'information génétique s'est traduite sous forme de protéine, elle ne peut plus en (res)sortir» [3]. De

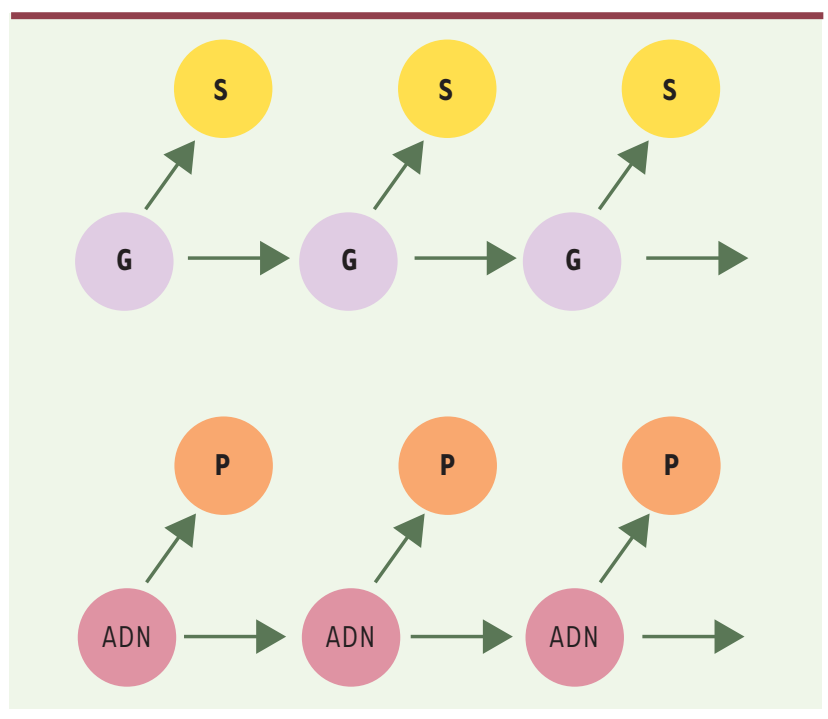

Figure 1. La théorie de Weismann. August Weismann prône la séparation fondamentale entre germen $(G)$ et soma (S), le germen étant à l'origine du soma et transmis de génération en génération; à l'inverse, il n'y a pas de contribution somatique à la lignée germinale. II existe ainsi une opposition entre la continuité transgénérationnelle de la lignée germinale et la discontinuité (impasse) de la lignée somatique. Cette figure (d'après [1]) illustre également l'isomorphisme existant entre le weismannisme [2] et le dogme central de la génétique moléculaire, proposé par Crick [3]. P : phénotype. même, Weismann défend la continuité de la lignée germinale de génération en génération, et la discontinuité de la lignée somatique; pour aller plus loin, le weismannisme défendrait même une autonomie des processus de l'hérédité (lignée germinale vers lignée germinale, ou génotype vers génotype) par rapport à ceux du développement (lignée germinale vers lignée somatique, ou génotype vers phénotype). Selon certains, d'ailleurs, les processus de transmission génétique pourraient être étudiés sans que soient pris en compte les problèmes, beaucoup plus complexes, de l'expression des gènes, et le weismannisme explique comment une telle argumentation a pu se développer $[6,9]$.

La biologie de la fin du xx siècle a vu l'émergence de différents projets synthétiques, réunissant notamment génétique et développement, évolution et développement, ainsi que plusieurs autres associations «évo-dévo impliquant des disciplines telles que la biologie cellulaire, la biochimie et même l'écologie. Il est probable que c'est la tentative de synthèse de la génétique et du développement qui a été la plus réductionniste, en raison de sa forte dépendance au weismannisme dans tout ce qui a concerné la construction de modèles, la conduite des expériences et l'interprétation des résultats: si les gènes constituent l'unique principe causal possible, ils deviennent, de fait, la première explication possible du développement. II n'est pas surprenant, dans ces conditions, que les visions moins dépendantes du weismannisme soient régulièrement en désaccord avec les interprétations de la génétique du développement.

\section{Considérer le développement comme un processus d'hérédité...}

Prenons des phénomènes typiquement qualifiés d'épigénétiques dans la littérature. La méthylation, qui induit une modification covalente des nucléotides, en est probablement le paradigme, bien que des protéines de liaison à l'ADN telles que les facteurs de transcription, des protéines chromosomiques comme les histones, et des structures encore plus complexes comme les protéines du groupe Polycomb constituent également des «systèmes de modification de la chromatine » $[7,10]$.

Deux précisions sont importantes à propos des systèmes de modification de la chromatine: d'une part, leurs effets sont dépendants de l'ADN ; d'autre part, leur étude a principalement pour objectif de comprendre les processus d'établissement, de régulation, de maintien et de transmission des différents états des cellules au sein d'organismes pluricellulaires. L'épigénétique concerne donc l'ensemble des voies par lesquelles les états cellulaires (au cours de la différenciation comme après la différenciation) sont établis au cours du développement, en fonction d'un contrôle épigénétique de l'expression génique comme des états cellulaires soumis à une hérédité cellulaire. Ainsi, Holliday définit l'épigénétique comme «l'étude des modifications de l'expression des gènes intervenant dans des 
organismes composés de cellules différenciées, et de l'hérédité, au cours de la mitose, de profils donnés d'expression génique » $[11]^{3}$. Le développement est donc appréhendé en termes de mise en place, maintien et transmission de variations épigénétiques au sein et parmi les lignées cellulaires. Mais cette interprétation du développement en termes d'hérédité cellulaire est encore dépendante de la relation inscrite par le weismannisme entre la génétique et l'épigénétique: de nouveau, tout processus causal ne pouvant être rattaché à la (variation de la) séquence nucléotidique relève de l'épigénétique, et demeure d'une importance toute relative en raison même du fait qu'il ne concerne pas le gène.

\section{...et, inversement, l’hérédité comme un processus développemental}

Une tradition très différente de la recherche en épigénétique concerne les interactions épigénétiques, «non génétiques», observées, au-delà du niveau moléculaire, au cours du développement. Le travail des embryologistes sur la morphogenèse (c'est-à-dire le processus développemental à l'origine de la formation des tissus et de la structure des organes), de même que les phénomènes décrits par C.H. Waddington, appartiennent à cette tradition.

Newman et Müller illustrent cette vision en décrivant les mécanismes épigénétiques comme des «facteurs à l'origine de la création de caractères morphologiques », ce qui est une vue plus large que celle apportée par le weismannisme, puisque les facteurs à l'origine de la création de caractères morphologiques ne sont plus nécessairement des gènes [12]. Comme chez Waddington, les phénomènes épigénétiques proviennent d'interactions «phénotypiques » prenant place à un niveau supérieur au niveau moléculaire, même s’il est clair que des mécanismes moléculaires interviennent également dans la création des caractères phénotypiques. Dans la théorie de l'assimilation génétique de Waddington, les modifications phénotypiques surviennent directement en réponse à des perturbations de l'environnement, comme dans ses fameuses observations sur les cals d'autruche ${ }^{4}$; le phénotype est ensuite génétiquement assimilé, par le biais d'une mutation de modificateurs des déterminants génétiques des caractères qui interagissent de façon phénotypique pour induire le changement initial. Newman et Müller parlent quant à eux de phénomènes «prémendéliens », dans lesquels une série d'interactions de tissus ou de cellules avec leur environnement physicochimique pourraient donner naissance à de nouveaux types morphologiques.

${ }^{3}$ Sa limitation de la définition de l'épigénétique à l'hérédité « au cours de la mitose » traduit probablement le fait que les modifications de la chromatine sont fréquemment observées dans le développement somatique, par opposition à l'hérédité au cours de la méiose.

${ }^{4}$ Waddington observa que les cals, normalement formés dans les zones de peau régulièrement soumises à la friction, sont observables dès la naissance chez l'autruche, au niveau des zones qui seront effectivement en contact répété avec le sol. II émit l'hypothèse selon laquelle ce caractère, acquis normalement au cours de la vie pouvait, chez l'autruche, être devenu «partie intégrante du génome» au cours de l'évolution, c'est-à-dire avoir fait l'objet d'une «assimilation génétique » (note du traducteur).
Bien que les composants de ces interactions aient évidemment un fondement génétique, l'émergence de nouveaux caractères ne nécessite pas la sélection de gènes favoris: les gènes jouent un rôle après la création épigénétique du caractère, afin de l'intégrer et de le stabiliser dans le génome. Une séquence différente est ainsi proposée dans l'ordre d'intervention des gènes et des mécanismes épigénétiques au cours du développement: comme dans l'assimilation génétique de Waddington, les caractères apparaissent d'abord, puis sont stabilisés.

Newman et Müller estiment que « différents processus épigénétiques ont prévalu à différentes étapes de l'évolution morphologique, et que les formes comme les caractères adoptés par les organismes pluricellulaires ont en grande partie pour origine .... [des interactions tissulaires et physicochimiques conditionnelles et non programmées de]... ces mécanismes » [12]. L'hérédité est alors plutôt interprétée sous l'angle du développement, comme un stade au cours d'une évolution fondamentalement dirigée par une origine épigénétique des caractères, et non par une variation de séquence génomique puis un contrôle épigénétique de l'expression génique. Et si les gènes interviennent avant les interactions épigénétiques dans l'émergence des caractères, ce n'est pas comme déterminants de cette émergence.

Cette vision des choses est explicitement antiweismannienne: les auteurs regrettent ainsi que «la notion d'un phénotype morphologique déterminé par le génotype soit admise dans toutes les visions concurrentes .... [de l'évolution des caractères]. Ce dogme n'est pas non plus remis en cause en biologie du développement, une discipline dont on dit qu'elle est l'étude des «programmes génétiques » à l'origine de la création de la structure des organismes et de la forme des organes » [12]. Mais la logique attractive du weismannisme moléculaire demeure: si des interactions épigénétiques entre tissus conduisent à l'émergence de nouveaux caractères, le fait que ces tissus aient des déterminants génétiques (et des déterminants moléculaires épigénétiques) avant de donner naissance à de nouveaux caractères pouvait réduire la théorie de Newman et Müller à une simple interprétation compatible avec le weismannisme, dans laquelle chaque «création » épigénétique d'un caractère nécessite une détermination génétique préalable. Pour réfuter cette interprétation simpliste de leur théorie, Newman et Müller ont souligné que leur travail concernait l'évolution des caractères morphologiques, ce qui signifie que l'argument weismannien doit pouvoir être validé jusque dans l'émergence de tous les caractères, c'est-à-dire à l'origine de la vie; cela reviendrait à partir du principe, qui relève de la théorie la plus extrême d'un monde originel constitué d'ARN, que la vie aurait pour origine un simple gène de ribozyme. Une autre solution est d'envisager que l'origine des premiers caractères ne relève pas du gène dans ses acceptions mendélienne ou moléculaire. En d'autres termes, les arguments ramenant vers le concept weismannien du gène unique cause, même indirecte, de l'émergence d'un caractère ne tiennent plus si le gène ne se situe pas à l'origine de la vie. 
II n'est bien sûr pas question, ici, de régler la question de l'origine de la vie. II se trouve seulement que les différentes manières de voir l'épigénétique, au niveau moléculaire ou non, dépendent toutes de la dichotomie fondamentale entre l'hérédité et le développement existant au sein du weismannisme. Que l'on veuille penser le développement comme une sorte d'hérédité, ou l'inverse, le weismannisme est omniprésent. Or I'on peut imaginer une autre façon de voir les choses, en s'affranchissant de cette théorie [13-16].

\section{Voir l'hérédité et le développement comme deux phénomènes entrelacés}

Malgré sa domination, le weismannisme ne peut rendre compte de tous les phénomènes épigénétiques : certains montrent que le flux d'information biologique peut aussi être à destination, et pas seulement en provenance, de l'ADN ; d'autres suggèrent l'existence d'une hérédité «somatique » indépendante de l'hérédité strictement dictée par la séquence de I'ADN. Mais au-delà même de cette incapacité à expliquer l'ensemble des phénomènes épigénétiques, il y a le fait que la variété des

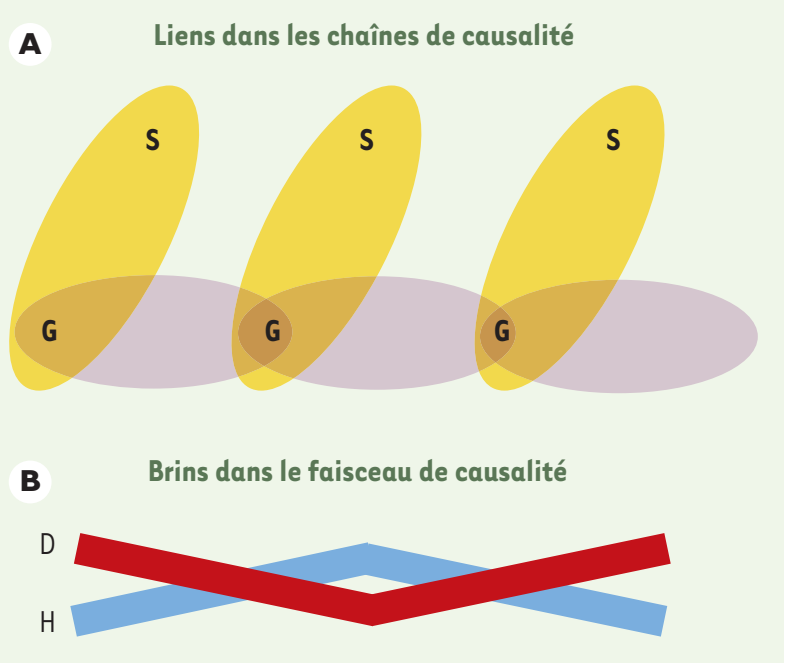

Figure 2. Modèles de causalité biologique: la chaîne et la corde. A. Ce modèle considère les causes et les effets comme des événements liés au sein d'une chaîne de causalité. Cette chaîne représente la continuité génétique (le germen, G), tandis que chaque lien qui en émerge latéralement représente, pour chaque génération, le soma (S), déterminé par la séquence génétique. La discontinuité des soma entre générations illustre pleinement la vision weismannienne de la causalité. B. Ce modèle de la corde, point de vue alternatif de la chaîne, considère l'hérédité et le développement comme des processus qui s'étendent à travers les cycles cellulaires et les générations. Ces processus se distinguent par le type de continuité assurée plutôt que par le fait que l'un, mais pas l'autre, serait discontinu: le développement (D), qui se retrouve aussi être un phénomène continu à travers le cycle de la vie, est ainsi représenté s'entrelaçant avec l'hérédité $(H)$. mécanismes épigénétiques permet de concevoir l'hérédité sous l'angle du développement, et inversement. Le weismannisme, d'ailleurs, n'interdit pas cela : il effectue seulement une séparation entre deux processus qui n'ont en commun, finalement, que d'être tous deux dirigés par les gènes.

Si le weismannisme décrit la relation germen/soma ou génotype/phénotype comme une succession de liaisons au sein d'une chaîne de causalité, une autre façon de voir les choses est d'utiliser la métaphore de la corde [17] (Figure 2), c'està-dire un entrecroisement de deux processus: l'hérédité et le développement sont les brins de cette corde et en occupent, I'un comme l'autre, toute la longueur. Mais si l'hérédité et le développement constituent les brins de la corde, que représente la corde elle-même? La réponse peut être: la reproduction. Celle-ci implique un entrelacement des processus de propagation génétique et d'émergence développementale et, tandis que la métaphore de la chaîne suggère que la reproduction est un événement ou une connexion unique entre parents et descendance, la métaphore de la corde suggère qu'elle est un processus étendu dans le temps.

Comment voir la reproduction comme un phénomène intervenant au cours de la vie d'une entité biologique plutôt que comme un événement de fusion gamétique ou nucléaire, ou de fission cellulaire? La reproduction, dans sa plus simple expression, est la multiplication d'entités s'accompagnant d'un recoupement physique d'éléments entre parents et descendance [13, 18] : différents éléments des parents (à un certain moment) deviennent éléments de la descendance (à un autre moment), la reproduction ne consistant donc plus en la simple transmission ou copie d'une forme, mais en un flux de matière. Certains des éléments parentaux transmis doivent avoir une caractéristique particulière, celle d'être des mécanismes du développement, pouvant acquérir la capacité de se reproduire. La reproduction devient alors la multiplication d'entités au cours de laquelle certains des éléments transmis à la descendance lui confèrent une capacité de développement, et donc de reproduction. Il s'agit là d'une séquence récurrente, qui illustre assez bien l'image de l'entrecroisement entre hérédité et développement au cours de la reproduction. L'hérédité est la corrélation entre parents et enfants liée à la reproduction, la reproduction transfère la capacité de se développer et le développement permet la reproduction.

\section{Le « concept reproducteur 》}

Différents points méritent d'être abordés pour valider l'analyse abstraite des processus de reproduction, d'hérédité et de développement réalisée à travers le concept reproducteur.

Un premier point concerne le recoupement matériel. N'importe quel processus de reproduction, tel qu'il vient d'être défini, nécessite l'intervention d'une hérédité dite «épigénétique»: puisqu'aucun processus de reproduction ne peut résulter d'un 
simple flux de gènes, d'autres éléments doivent être transmis afin de fabriquer une nouvelle entité capable de se développer. Jablonka et Lamb ont décrit trois catégories de systèmes d'hérédité épigénétique: les systèmes de modification $(\rightarrow) \mathrm{m} / \mathrm{s}$ 2005, $n^{\circ} 4$, p. 377 de la chromatine (mentionnés plus haut), les systèmes d'hérédité « métabolique » à l'équilibre et les systèmes d'hérédité structurale [10]. Le plus connu des systèmes d'hérédité structurale n'impliquant pas de marquage de la chromatine est celui décrit par Sonneborn chez la paramécie $(\rightarrow)$.

Les systèmes à l'équilibre impliquent, quant à eux, une transmission de profils métaboliques grâce à l'équilibre chimique qu'ils produisent. Leur perpétuation dépend de la façon dont les métabolismes sont dupliqués au cours de la division cellulaire: dans la mesure où les métabolismes sont autocatalytiques et fluides [19], leur division globale en deux parties séparées dans l'espace permet une transmission de l'état d'équilibre à chacune des cellules filles; une «mutation» de cet état d'équilibre en raison d'une perturbation environnementale peut aboutir à un nouvel état d'équilibre, lui aussi transmissible. On voit que ces deux types d'hérédité, structurale et métabolique, impliquent l'existence d'un recoupement matériel d'éléments, aussi bien épigénétiques que génétiques, au cours de la reproduction. Si l'un comme l'autre de ces types d'hérédité jouent un rôle développemental dans la mesure où ils entraînent l'acquisition de la capacité à se reproduire, alors ce sont des mécanismes du développement tels que définis plus haut et le système qui les contient est « reproducteur » [13-16, 18].

Un deuxième point à souligner concernant la validité scientifique du concept reproducteur est son entière indépendance vis-à-vis des gènes comme des acides nucléiques, une indépendance qui permet d'analyser le problème de la transition évolutive (c'est-à-dire de l'origine évolutive des nouveaux niveaux d'organisation biologique) sans faire référence à l'existence de «réplicateurs » [15]: dans cette nouvelle vision, la transition évolutive ne nécessite pas que les gènes aient existé avant les systèmes capables de subir des variations héréditaires, au contraire de ce que réclame la théorie de l'évolution, non circulaire, de l'origine des gènes. Le concept reproducteur autorise ainsi l'évolution d'une unité de reproduction sans le concours des gènes, tandis que la vision weismannienne nécessite la création spontanée des premiers réplicateurs.

Une autre question d'importance est d'ajuster la représentation de l'hérédité épigénétique dans ses relations avec la reproduction et les processus d'hérédité conventionnelle, c'est-à-dire génétique. Si la reproduction biologique est un type de multiplication impliquant le recoupement partiel de mécanismes développementaux, l'hérédité est un processus de reproduction impliquant le recoupement partiel de mécanismes développementaux évolués. En d'autres termes, pour constituer un système d'hérédité, les éléments appartenant aux mécanismes développementaux doivent avoir suffisamment évolué pour pouvoir assurer, justement, leur rôle dans le développement. II n'est pas fait explicitement référence aux gènes dans cette définition: l'évolution a pu intervenir sans le concours d'aucun système d'hérédité, par le biais d'une héritabilité résultant simplement de ce recoupement matériel. L'évolution adapatative se sera par la suite affinée dès lors que les mécanismes développementaux auront eux-mêmes évolué en termes de spécialisation, de partage des tâches au cours du développement, de diversification écologique, et de stabilisation des nouveaux niveaux d'organisation grâce à une séparation d'avec les éléments peu coopératifs. Une autre évolution expérimentée par ces mécanismes développementaux a été leur organisation au sein d'une structure très spécialisée dotée d'un codage, à même de leur fournir une certaine autonomie. En général, les systèmes autonomes biologiques se sont, à certains égards et à un certain degré, affranchis des contraintes chimiques, une propriété qui peut être nommée «liberté stœchiométrique»; l'exemple le plus évident de ce type de système de codage est celui du codage génétique fondé sur la séquence d'ADN.

De l'ensemble de ces points commence à émerger une image. La reproduction est le type de processus le plus général dans lequel l'hérédité et le développement s'entrecroisent. Sa structure fondamentale résulte du recoupement matériel d'éléments ayant une organisation autocatalytique et autopoḯtique (organisation et maintien autonomes) [16, 19]. L'hérédité est, de son côté, un cas particulier de processus reproductif dans lequel les mécanismes développementaux ont évolué afin de remplir des fonctions développementales. L'hérédité génétique est, quant à elle, un cas particulier d'hérédité dans lequel les mécanismes développementaux ont évolué jusqu'à atteindre un niveau «codant» d'organisation. Sous cet angle, l'épigénétique décrit une série de systèmes d'hérédité non génétiques. Mais il n'est plus indispensable de définir l'épigénétique par rapport à ce qui est génétique: de fait, l'existence de mécanismes évolués d'hérédité génétique n'est plus nécessaire à l'existence de l'hérédité épigénétique. Les relations entre reproduction, épigénétique et hérédité génétique sont définies en termes de relations entre le général et le particulier, et non plus en termes de relations de causalité entre les types de systèmes (hormis leurs relations de causalité au cours de l'évolution). Dans ce sens, le concept reproducteur donne une interprétation moléculaire et physicochimique aux notions véhiculées par l'épigénétique, utilisées pour décrire différents aspects d'un processus en termes d'hérédité ou de développement, hors de toute référence à une quelconque hiérarchisation de niveaux d'organistion biologique.

\section{Conclusions : quelle est la signification d'《épi 》 dans « épigénétique »?}

Du point de vue du weismannisme, tout ce qui est «hors» de la séquence de l'ADN ou de ses variations est «épigénétique ». Cette définition est si large qu'elle rend virtuellement impossible toute tentative de classement théorique des phénomènes épigénétiques ou, plus simplement, toute recherche de simples règles d'organisation. Dans le weismannisme, les gènes sont monar- 
ques au royaume du développement; I'ironie est qu'en faisant du gène la causalité de tout, le gène seul ne peut rien expliquer. Nous avons vu que les concepts sur l'épigénétique peuvent être classés en deux catégories : ceux traitant de l'hérédité comme du développement, et ceux traitant du développement comme de l'hérédité. L'idée est de trouver une théorie intermédiaire permettant d'expliquer certains phénomènes frappants sans avoir recours à une explication développementale unique - les gènes - mais sans non plus faire référence à un concept holistique inexploitable. Cette tentative se trouve hélas entravée par la définition weismannienne de ce qui est épigénétique.

Cette autre façon de voir les choses peut être le concept reproducteur: le préfixe «épi » s'entend alors de façon quasiment littérale, dans la mesure où nous sommes dans la vision d'un processus physique plutôt que formel ou structural. Est épigénétique ce qui est effectivement «sur » les gènes : systèmes de modification de la chromatine ou de méthylation de l'ADN, facteurs de transcription, histones... Bien sûr, l'héritabilité de ces systèmes ne repose pas uniquement sur les gènes: ils ne sont transmis que dans le contexte d'une hérédité structurale, ou à l'équilibre, de la machinerie moléculaire nécessaire à la (dé)méthylation de l'ADN dans la descendance. Ces derniers mécanismes ne sont quant à eux pas obligatoirement à classer dans l'hérédité «épigénétique » : du point de vue du concept reproducteur, il s'agit simplement d'hérédité. Le concept reproducteur qualifie ainsi moins facilement un événement d'épigénétique, favorisant en cela les interactions entre généticiens, épigénéticiens et autres personnes intéressées par les origines et l'évolution des processus de la reproduction et du développement. $\diamond$

\section{SUMMARY}

What is « epi » about epigenetics?

What counts as epigenetic depends on what counts as genetic. It is argued that Weismannism, the doctrine of genetic continuity and somatic discontinuity, is the basis for an overly inclusive concept of epigenetics as every inherited resource «beyond the genes ». An alternative theoretical perspective, the «reproducer» concept, facilitates analysis of multiple inheritance systems without labeling all non-genetic inheritance « epigenetic ». $\diamond$

\section{RÉFÉRENCES}

1. Maynard Smith J. The theory of evolution, $3^{\text {rd }}$ ed. Middlesex: Penguin, 1975.

2. Weismann A. Das Keimplasma, Eine theorie der Vererbung. Jena: Gustav Fischer, 1892.

3. Crick F. On protein synthesis. Symp Soc Exp Biol 1958; 12 : 138-63.

4. Winther R. August Weismann on germ-plasm variation. J Hist Biol $2001 ; 34$ 517-55.

5. Griesemer J, Churchill FB. Weismann, August Friederich Leopold. In : Pagel M, ed. Encyclopedia of evolution, vol. 2. New York : Oxford University Press, 2002 : 1149 51.

6. Griesemer J. Reproduction and the reduction of genetics. In : Beurton P, Falk $\mathrm{R}$, Rheinberger $\mathrm{HJ}$, eds. The concept of the gene in development and evolution, historical and epistemological perspectives. New York : Cambridge University Press, $2000: 240-85$.

7. Bestor TH, Chandler VL, Feinberg AP. Epigenetic effects in eukaryotic gene expression. Dev Genet $1994 ; 15: 458-62$.

8. Griesemer JR, Wimsatt WC. Picturing Weismannism: a case study of conceptual evolution. In : Ruse M, ed. What the philosophy of biology is. Essays for David Hull. Dordrecht: Kluwer Academic Publishers, 1989 : 75-137.

9. Griesemer J. Tracking organic processes: representations and research styles in classical embryology and genetics. In : Maienschein J, Laubichler M, eds. From embryology to evo-devo. London : MIT Press, 2005 (sous presse).

10. Jablonka $\varepsilon$, Lamb M. Epigenetic inheritance and evolution. Oxford: Oxford University Press, 1995.

11. Holliday R. Epigenetics: an overview. Dev Genet 1994 ; 15 : 453-7.

12. Newman SA, Müller GB. Epigenetic mechanisms of character origination. J Exp Zool $2000 ; 288: 304-17$

13. Griesemer J. The units of evolutionary transition. Selection $2000 ; 1: 67-80$.

14. Griesemer J. Limits of reproduction: a reductionistic research strategy in evolutionary biology. In : Van Regenmortel MHV, Hull D, eds. Promises and limits of reductionism in the biomedical sciences. Chichester: John Wiley and Sons Ltd, $2002: 211-31$.

15. Maynard Smith J, Szathmáry $\varepsilon$. The major transitions in evolution. Oxford: W.H. Freeman Spektrum, 1995.

16. Varela F. Principles of biological autonomy. New York: Elsevier North Holland, 1979.

17. Venn J. The logic of chance. An essay on the foundations and province of the theory of probability, with especial reference to its logical bearings and its application to moral and social science, $2^{\text {nd }}$ ed. London : MacMillan, 1876.

18. Griesemer J. Development, culture and the units of inheritance. Philosophy of Science $2000 ; 67$ : S348-68.

19. Gánti T. The principles of life. New York: Oxford University Press, 2003.

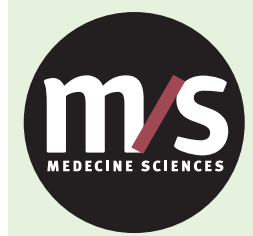

Tarifs d'abonnement M/S - 2006

Abonnez-vous

à Médecine/Sciences
$>$ 1985-2005, depuis 20 ans, grâce à $\mathrm{m} / \mathrm{s}$, vous vivez en direct les progrès des sciences biologiques et médicales

TIRÉS À PART

J. Griesemer

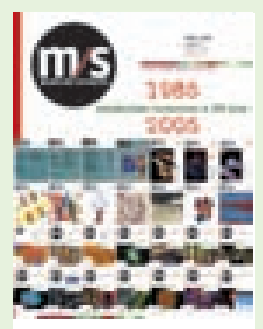

